Case Report

\title{
A Case Study of Intractable Vomiting with Final Diagnosis of Neuromyelitis Optica
}

\author{
Rachel Bramson ${ }^{1,2}$ and Angela Hairrell ${ }^{1}$ \\ ${ }^{1}$ College of Medicine, Texas A\&M Health Science Center College of Medicine, Bryan, TX 77807, USA \\ ${ }^{2}$ Family Medicine, Scott and White University Clinic, College Station, TX 77845, USA \\ Correspondence should be addressed to Rachel Bramson; bramson@medicine.tamhsc.edu
}

Received 30 June 2015; Accepted 21 September 2015

Academic Editor: Denis A. Cozzi

Copyright ( 2015 R. Bramson and A. Hairrell. This is an open access article distributed under the Creative Commons Attribution License, which permits unrestricted use, distribution, and reproduction in any medium, provided the original work is properly cited.

\begin{abstract}
This case study presents a patient living in a suburban/rural community who received appropriate referral to secondary and tertiary care for nausea and vomiting, accompanied by waxing and waning neurological symptoms, yet proved difficult to diagnose. This patient is presented to draw attention to a rare neurological disorder which should be included in the differential diagnosis of nausea and vomiting with some key neurological complaints, even in the absence of physical findings.
\end{abstract}

\section{Introduction}

When a previously healthy adolescent presents with nausea and vomiting, the most common diagnoses are viral gastroenteritis or pyelonephritis. Vague waxing and waning neurological symptoms in an adolescent may be attributed to stress or developmental issues. Here we present a patient living in a suburban/rural community who received appropriate referral to secondary and tertiary care, yet proved difficult to diagnose. This patient is presented to draw attention to a rare neurological disorder which should be included in the differential diagnosis of nausea and vomiting with some key neurological complaints, even in the absence of physical findings. Physical findings may develop late in the course of the disease making a heightened index of suspicion important for early diagnosis and treatment.

\section{Patient Presentation}

The patient is a 17-year-old African-American female, multisport athlete in 11th grade in a rural high school. She is the second of four children and lives at home with her parents and two siblings. She gets Bs and Cs in school and is extremely well-liked by teachers and fellow students. She presented to Urgent Care with abdominal pain, nausea, and vomiting with a $19 \mathrm{lb}$. unintentional weight loss over a three-month period and BMI of 20 . Her past medical history revealed normal development, immunizations being up to date, and pyelonephritis at age 14 for which she was hospitalized. She had no past surgeries and no history of alcohol, tobacco, or drug use. Her parents and siblings are healthy.

\section{Clinical Findings}

On initial presentation, the patient appeared healthy with no notable physical findings. She was discharged on Zofran and a clear liquid diet. On a follow-up two days later she had mild epigastric tenderness on physical exam and described a problem with shaky hands and back pain radiating into the right leg off and on, worse when she runs. The neurological exam was normal. CBC and CMP were normal with the exception of low WBC 3400 and sodium 134. TSH was low normal (0.5). On Beck's Anxiety Inventory she scored 11 (mild), and on Beck's Depression Inventory she scored 1 (negative).

\section{Timeline}

Due to the protracted course of this patient's illness and workup, Table 1 details her visits and care over a three-month period. The following is a brief summary of her clinical presentation. 


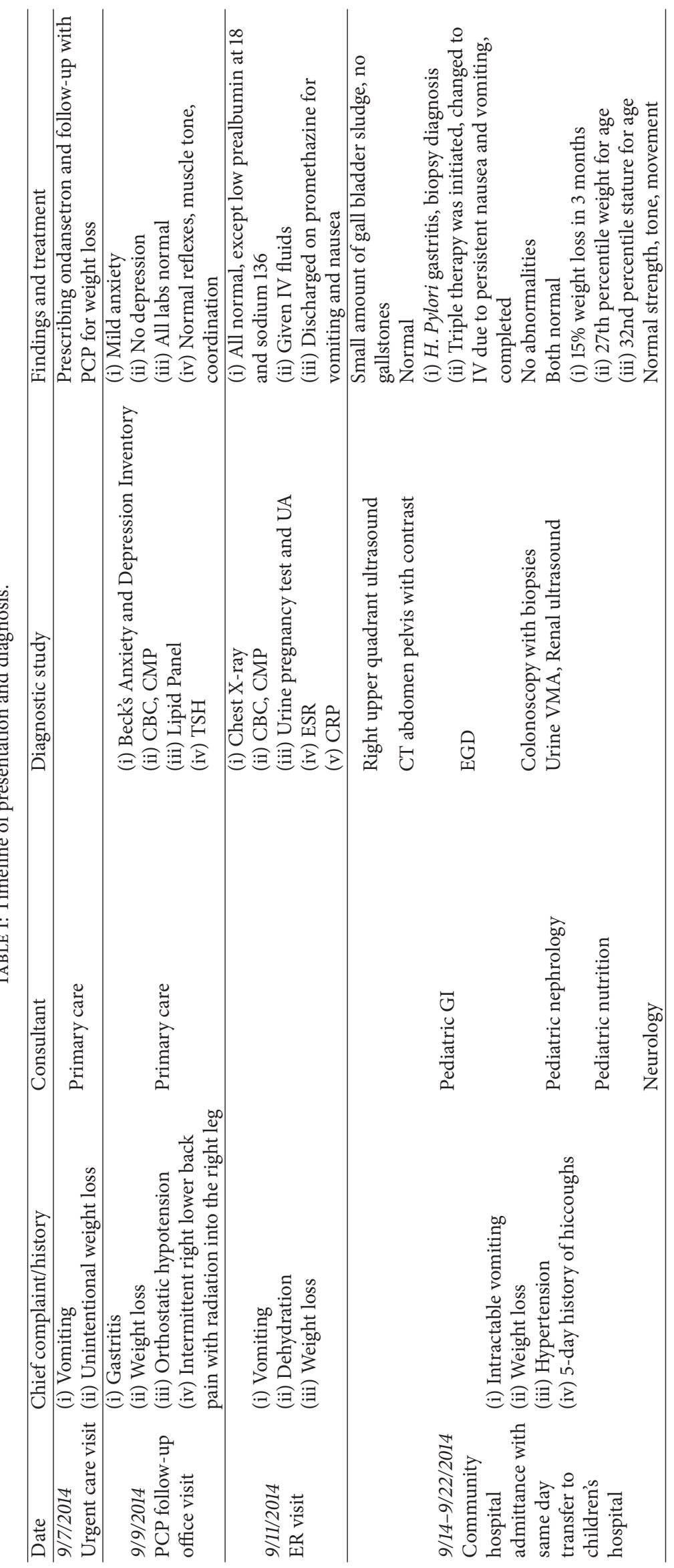




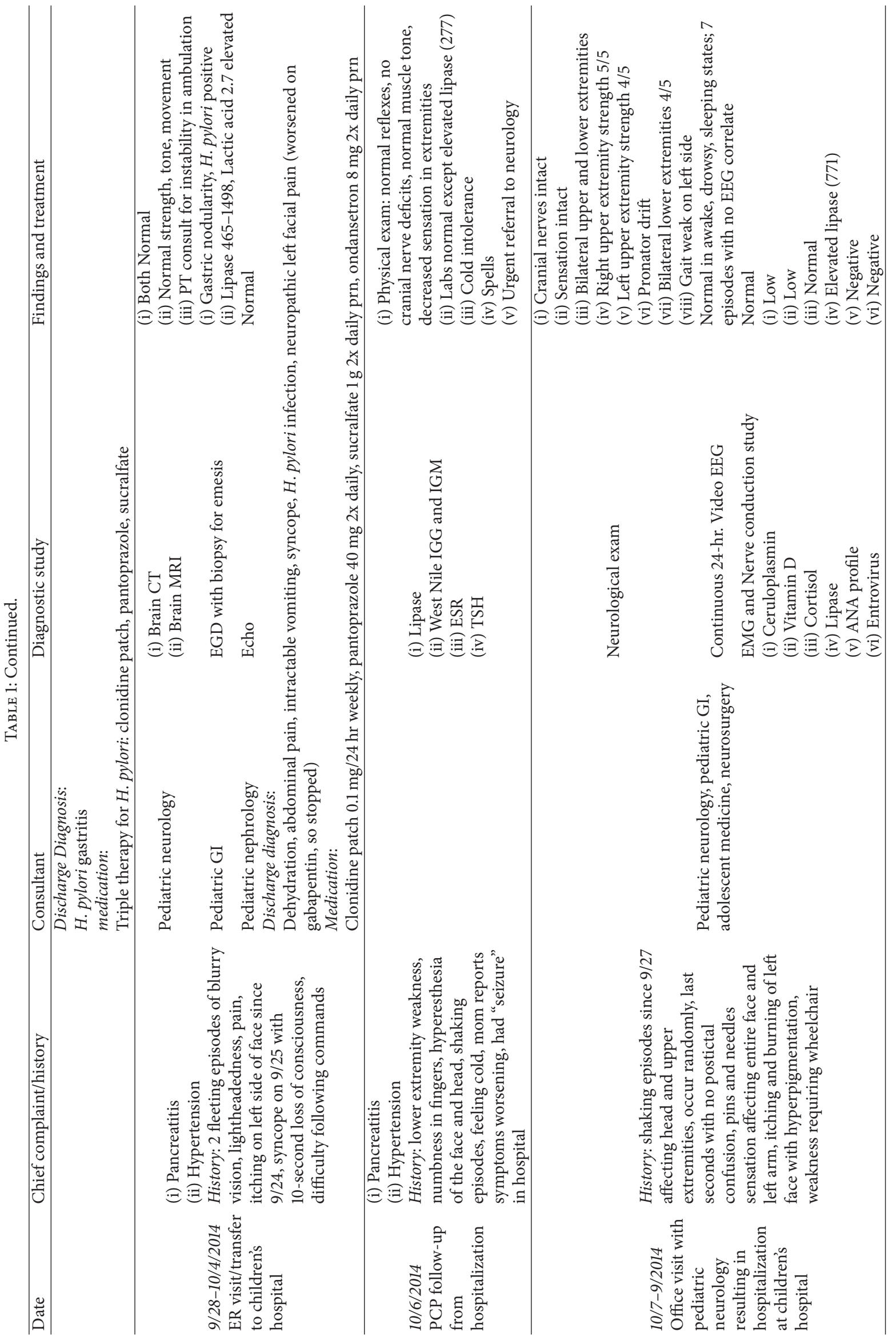




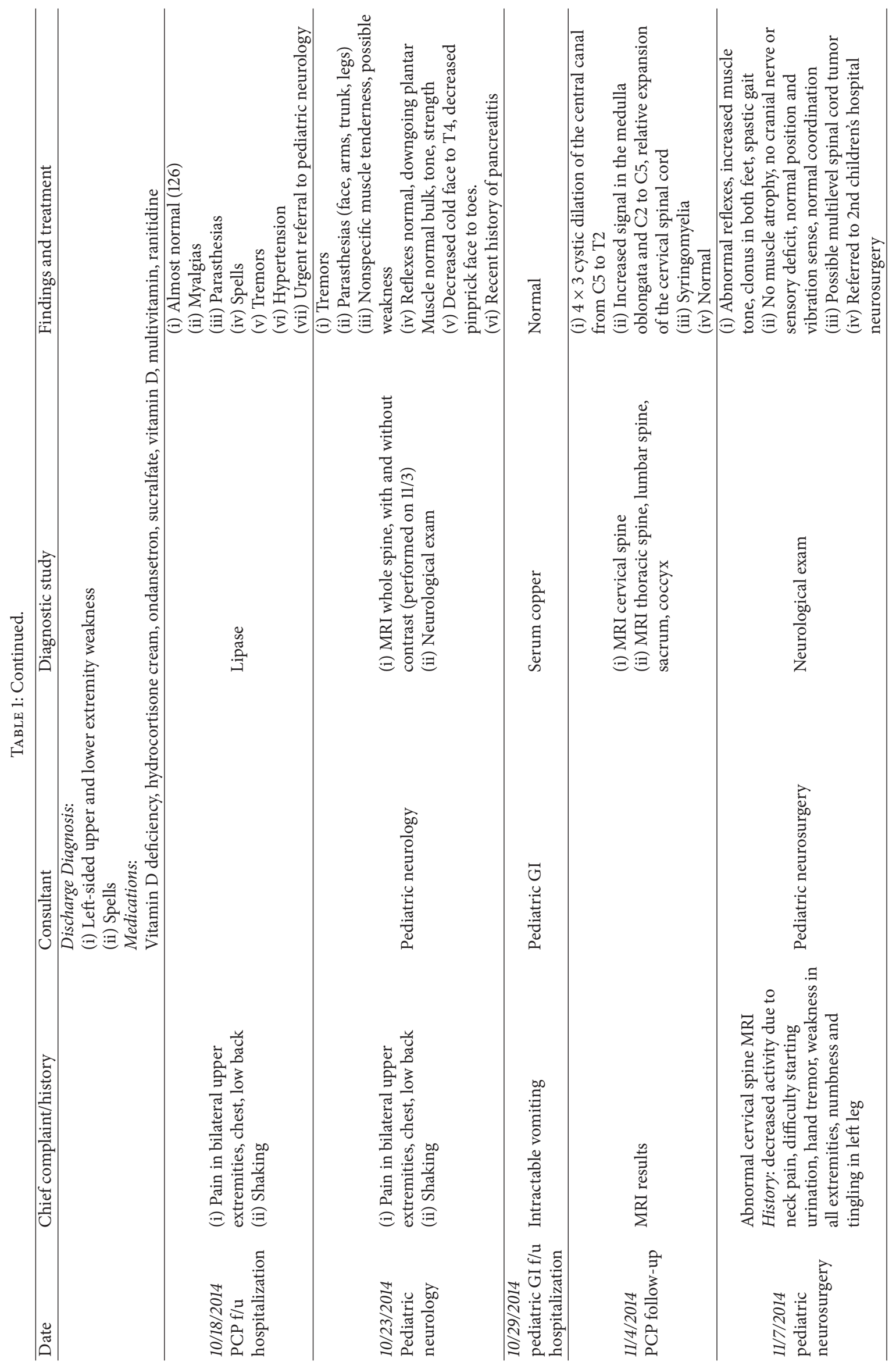




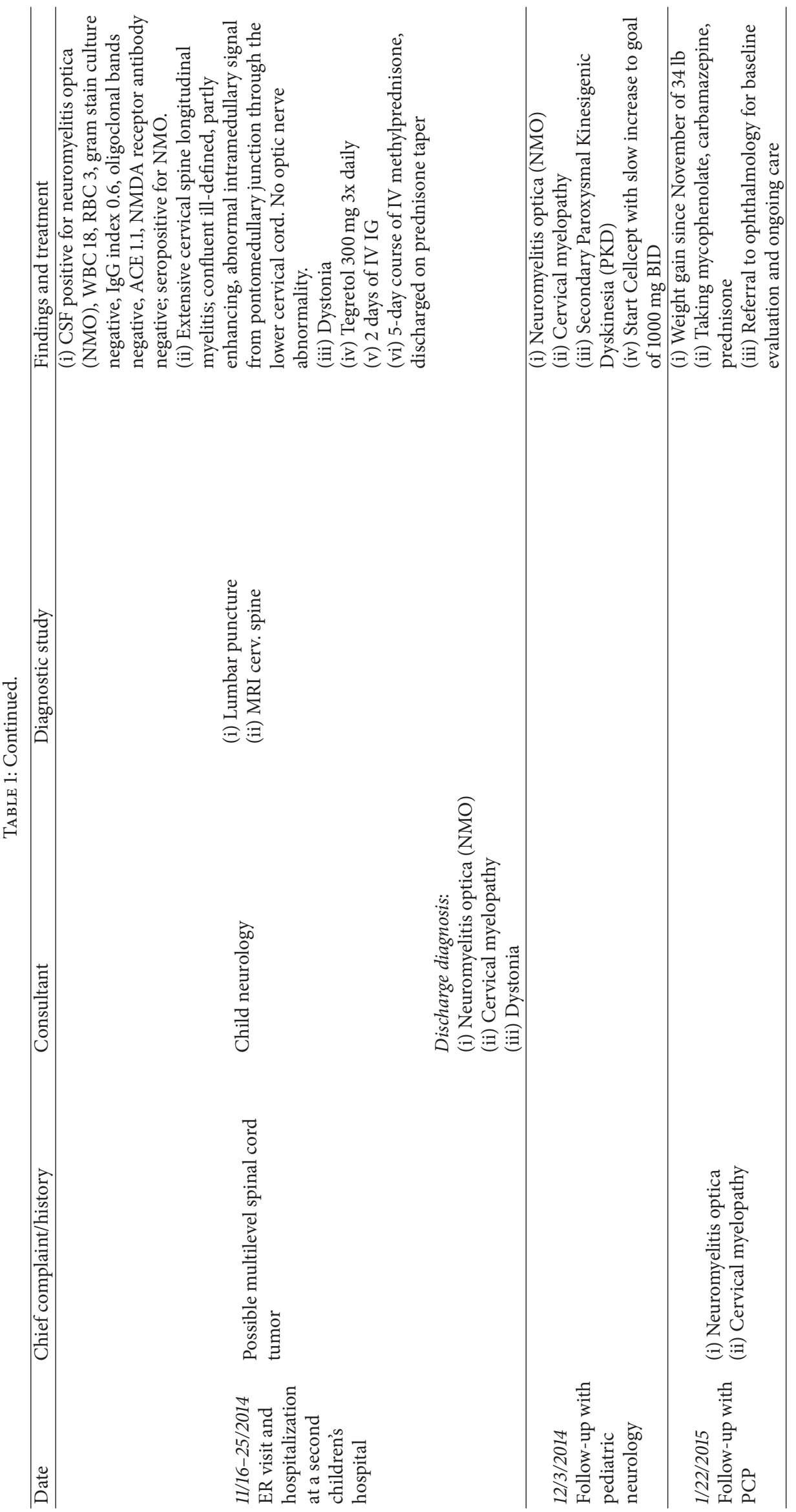


Two days after her initial presentation, she presented to the emergency room with vomiting and dehydration. Bloodwork and chest X-ray were unremarkable except for low prealbumin. She was treated with IV fluids and discharged on promethazine to relieve the nausea and vomiting. Three days later, she presented with intractable vomiting and a five-day history of hiccoughs. She was admitted to the local hospital and transferred the same day to the regional children's hospital. She was discharged eight days later with a biopsy-proven diagnosis of $H$. pylori gastritis and hypertension having had multiple imaging studies, upper and lower endoscopy, and renal and nutrition consultation for $15 \%$ weight loss in three months. Neurological exams revealed normal reflexes, strength, tone, coordination, and movement. Discharge medication included triple therapy for $H$. pylori, sucralfate, clonidine patch, and ondansetron.

Six days later, she presented to the emergency room and was transferred back to the regional children's hospital for pancreatitis and hypertension with additional history of fleeting blurry vision and pruritus of the left face. Workup included a neurology consult, brain imaging, EGD with biopsy, and echocardiogram, all unremarkable. Neuropathic facial pain was added to her diagnoses. Treatment continued unchanged.

In follow-up office visit two days later, the patient's mother insisted she was having lower extremity weakness, shaking episodes that looked like seizures, and hypersensitivity of the face and head. An urgent referral to neurology resulted in hospitalization at the regional children's hospital. Neurological exam showed slightly decreased strength of the left upper extremity and bilateral lower extremities, pronator drift, and gait weakness on the left side. Video EEG, EMG, and nerve conduction studies were unrevealing. Wilson's disease was ruled out. The patient was found to have a vitamin $\mathrm{D}$ deficiency, normal cortisol, and negative ANA.

Nine days later in follow-up with the primary care provider, tremors were witnessed and the patient had intense pain of the bilateral upper extremities, chest, and low back. Urgent referral to pediatric neurology resulted in an order for MRI of the whole spine with and without contrast which was performed eight days later. The next day, the radiology results were described to the patient (increased signal in the medulla oblongata, between $\mathrm{C} 2$ and $\mathrm{C} 5$, and possible diagnosis of syringomyelia). Three days later she saw a pediatric neurosurgeon who suspected a multilevel spinal cord tumor and referred her to a tertiary care children's hospital department of neurosurgery. Due to difficulty scheduling an outpatient consultation, the patient presented to the emergency room nine days later and was admitted for a possible multilevel spinal cord tumor.

\section{Outcome}

At the tertiary care children's hospital, CSF from lumbar puncture was NMO positive with $18 \mathrm{WBC}, 3 \mathrm{RBC}$, gram stain and culture negative, $\operatorname{IgG}$ index 0.6 , oligoclonal bands negative, ACE 1.1, and NMDA receptor antibody negative. MRI of the cervical spine revealed confluent ill-defined, partly enhancing, abnormal intramedullary signal from the pontomedullary junction through the lower cervical cord with no optic nerve abnormality (no syringomyelia or spinal cord tumor). She was also diagnosed with extensive cervical myelopathy and dystonia. She was started on carbamazepine, two days of IV IG, and five days of IV methylprednisone and discharged on a prednisone taper.

Eight days later she followed up with pediatric neurology and started mycophenolate with the goal of a $1000 \mathrm{mg}$ BID. She was noted to have Secondary Paroxysmal Kinesigenic Dyskinesia (PKD).

Six weeks later in a follow-up with her primary care physician, she had gained 34 pounds and was doing well on mycophenolate, carbamazepine, and prednisone.

\section{Patient Perspective}

Mother's perspective: I knew something was wrong with my daughter. It was frustrating to have to ask for testing. I am glad that we finally got a diagnosis. Now my daughter is much better.

\section{Discussion}

Physicians involved in the management of this patient were diligent in efforts to reveal the underlying cause of intractable nausea and vomiting. Several factors contributed to the difficulty of determining the correct underlying diagnosis. (1) Nausea and vomiting were the presenting problem for one month. (2) The patient's neurological symptoms presented late and were vague and diffuse with only subtle findings on physical exam. While her mother reported "shaking spells," the tremors observed appeared to be a stress response. (3) The normal CT and MRI of the brain were falsely reassuring that a demyelinating disorder or other neurological disease was not the underlying cause. (4) The patient developed neck pain late in the course of her illness. The combination of tremors, paresthesias, nonspecific muscle tenderness, and weakness led to whole spine MRI. The cervical spine MRI revealed the abnormal findings which allowed subsequent diagnosis.

This patient's diagnostic workup also demonstrates a critical point about interpretation of abnormal spinal cord images: a false diagnosis of spinal cord tumor can result in unnecessary biopsy of the spinal cord. Fortunately, the neurosurgeon referred the patient to a tertiary care setting where further evaluation resulted in the correct diagnosis and treatment.

Neuromyelitis optica (NMO) is an uncommon disease syndrome of the central nervous system (CNS) that affects the optic nerves and spinal cord. Individuals with NMO develop optic neuritis (pain in the eye and vision loss) and transverse myelitis (weakness, numbness, and sometimes paralysis of the arms and legs), along with sensory disturbances and loss of bladder and bowel control [1-5].

NMO is distinguished from multiple sclerosis by positive serum autoantibody NMO-IgG, which targets aquaporin 4 [6-11]. Over the last nine years, the aquaporin 4 serum test has allowed identification of a wider spectrum of clinical and radiological characteristics associated with NMO. 
In 2006 [3], diagnostic criteria were developed for a related clinical syndrome, NMOSD (NMO Spectrum Disorder). This requires NMO-IgG seropositive status in association with a limited form of NMO or a "signature clinical syndrome," such as intractable nausea, vomiting, or hiccoughs $[5,12,13]$.

This patient demonstrates several typical findings of NMO, presented here to encourage clinicians to identify this unusual constellation of symptoms. NMO should be considered in the differential for intractable nausea and vomiting [13]. Analysis of a multicenter study by Mealy et al. [14] provides the best current characteristics of patients with NMO and NMOSD. This study examined 187 patients who were diagnosed with NMO or NMODS at three nationally known medical centers distributed across the US.

Our patient shares several characteristics with patients in the multicenter study. As in other autoimmune disorders, females predominate $(6.5: 1)$ [14]. African Americans were overrepresented at $37 \%$ [14], while they represent only $13.2 \%$ of the US population [15]. Additionally, of those patients in the study with brain stem lesions ( $n=30 / 187), 80 \%$ ( $n=$ 24/30) were of African descent [14]. The disproportionate prevalence of NMO in African Americans and their increased incidence of brain stem lesions deserve further study. However, while our patient was only 17, the median age of onset in the study group was 40 years (range: $3-81$ ) [14].

Other common manifestations of NMO were also noted in this patient [5]. For example, most patients with transverse myelitis due to NMO have extensive longitudinal involvement ( $\geq 3$ vertebral segments), as did our patient. Additionally there are reported cases of generalized pruritus early in the disease $[9,16,17]$; this patient had focal pruritus of the left cheek which became hyperpigmented due to inflammation and scratching. This was the only clue to the itching she was experiencing. This patient also developed hypertension.

NMO has yet to receive funding for a national multicenter consortium; however, a five-year multicenter analysis in 2012 [14] revealed several key features of the disease that this patient demonstrates. Pediatricians and other primary care providers should be sensitized to the unusual presenting symptoms of NMO to optimize early detection and treatment. Early detection and treatment seem to improve prognosis and reduce relapse rate.

\section{Conflict of Interests}

The authors declare that there is no conflict of interests regarding the publication of this paper.

\section{References}

[1] Office of Communications and Public Liaison, National Institute of Neurological Disorders and Stroke, and National Institutes of Health, NINDS Neuromyelitis Optica Information Page, 2015, http://www.ninds.nih.gov/disorders/neuromyelitis_optica/neuromyelitis_optica.htm.

[2] D. M. Wingerchuk, W. F. Hogancamp, P. C. O’Brien, and B. G. Weinshenker, "The clinical course of neuromyelitis optica (devic's syndrome)," Neurology, vol. 53, no. 5, pp. 1107-1114, 1999.
[3] D. M. Wingerchuk, V. A. Lennon, S. J. Pittock, C. F. Lucchinetti, and B. G. Weinshenker, "Revised diagnostic criteria for neuromyelitis optica," Neurology, vol. 66, no. 10, pp. 1485-1489, 2006.

[4] M. A. Lana-Peixoto, "Devic's neuromyelitis optica: a critical review," Arquivos de Neuro-Psiquiatria, vol. 66, no. 1, pp. 120138, 2008.

[5] M. Matiello, A. Jacob, D. M. Wingerchuk, and B. G. Weinshenker, "Neuromyelitis optica," Current Opinion in Neurology, vol. 20, no. 3, pp. 255-260, 2007.

[6] B. G. Weinshenker and D. M. Wingerchuk, "The two faces of neuromyelitis optica," Neurology, vol. 82, no. 6, pp. 466-467, 2014.

[7] S. L. Galetta and J. Bennett, "Neuromyelitis optica is a variant of multiple sclerosis," Archives of Neurology, vol. 64, no. 6, pp. 901-903, 2007.

[8] D. M. Wingerchuk, V. A. Lennon, C. F. Lucchinetti, S. J. Pittock, and B. G. Weinshenker, "The spectrum of neuromyelitis optica," Lancet Neurology, vol. 6, no. 9, pp. 805-815, 2007.

[9] M. Muto, M. Mori, Y. Sato et al., "Current symptomatology in multiple sclerosis and neuromyelitis optica," European Journal of Neurology, vol. 22, no. 2, pp. 299-304, 2015.

[10] P. V. A. Lennon, D. M. Wingerchuk, T. J. Kryzer et al., "A serum autoantibody marker of neuromyelitis optica: distinction from multiple sclerosis," The Lancet, vol. 364, no. 9451, pp. 2106-2112, 2004.

[11] P. Huppke, M. Blüthner, O. Bauer et al., "Neuromyelitis optica and NMO-IgG in European pediatric patients," Neurology, vol. 75, no. 19, pp. 1740-1744, 2010.

[12] C. Trebst, S. Jarius, A. Berthele et al., "Update on the diagnosis and treatment of neuromyelitis optica: recommendations of the Neuromyelitis Optica Study Group (NEMOS)," Journal of Neurology, vol. 261, no. 1, pp. 1-16, 2014.

[13] M. Apiwattanakul, B. F. Popescu, M. Matiello et al., "Intractable vomiting as the initial presentation of neuromyelitis optica," Annals of Neurology, vol. 68, no. 5, pp. 757-761, 2010.

[14] M. A. Mealy, D. M. Wingerchuk, B. M. Greenberg, and M. Levy, "Epidemiology of neuromyelitis optica in the United States: a multicenter analysis," Archives of Neurology, vol. 69, no. 9, pp. 1176-1180, 2012.

[15] Centers for Disease Control and Prevention, "Minority health: Black and African American populations," 2015, http://www .cdc.gov/minorityhealth/populations/REMP/black.html.

[16] R. Govindarajan and E. Salgado, "What is the true clinicopathologic spectrum of neuromyelitis optica?" JAMA Neurology, vol. 70, no. 2, pp. 272-273, 2013.

[17] L. Elsone, T. Townsend, K. Mutch et al., "Neuropathic pruritus (itch) in neuromyelitis optica," Multiple Sclerosis, vol. 19, no. 4, pp. 475-479, 2013. 


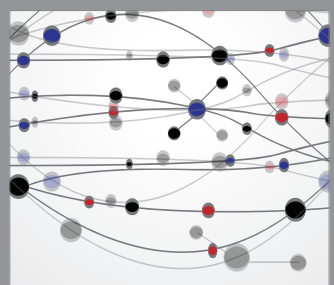

The Scientific World Journal
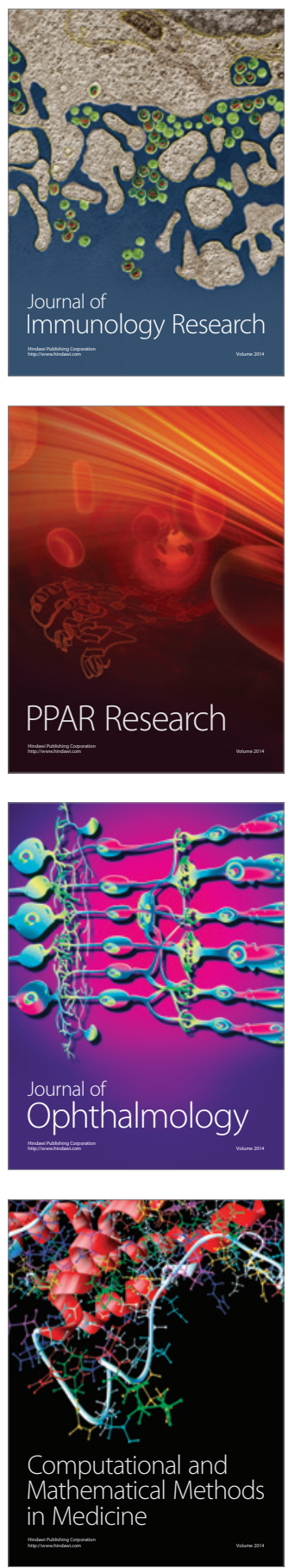

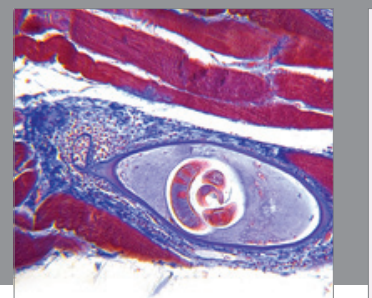

Gastroenterology

Research and Practice
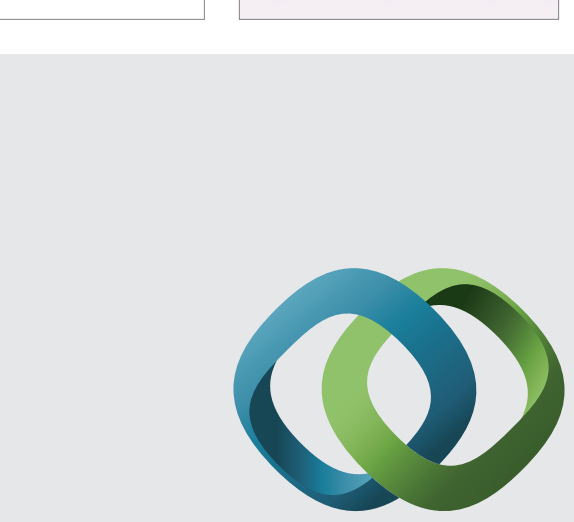

\section{Hindawi}

Submit your manuscripts at

http://www.hindawi.com
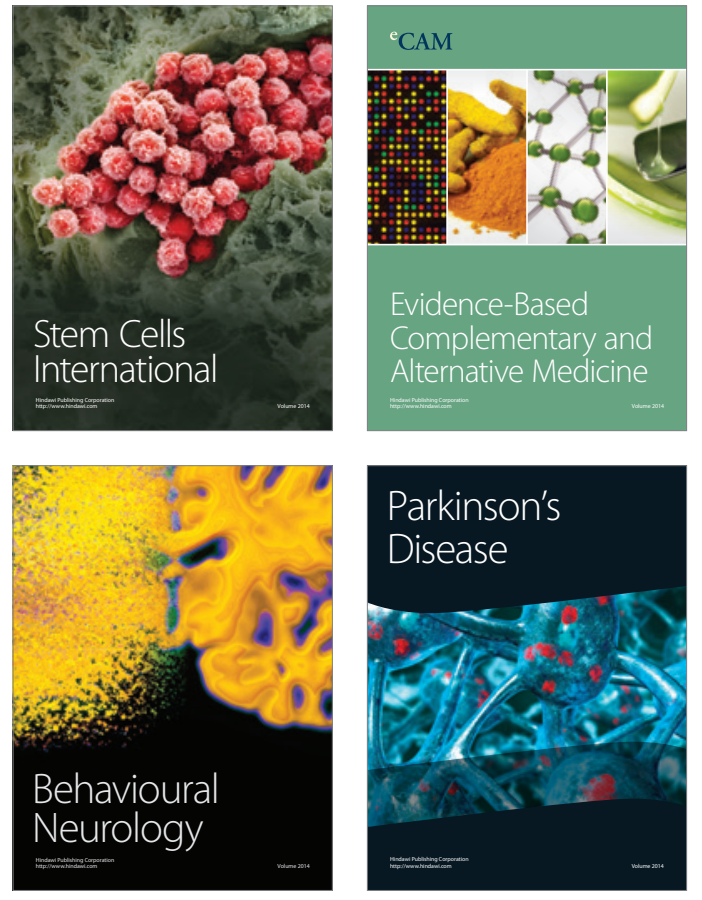
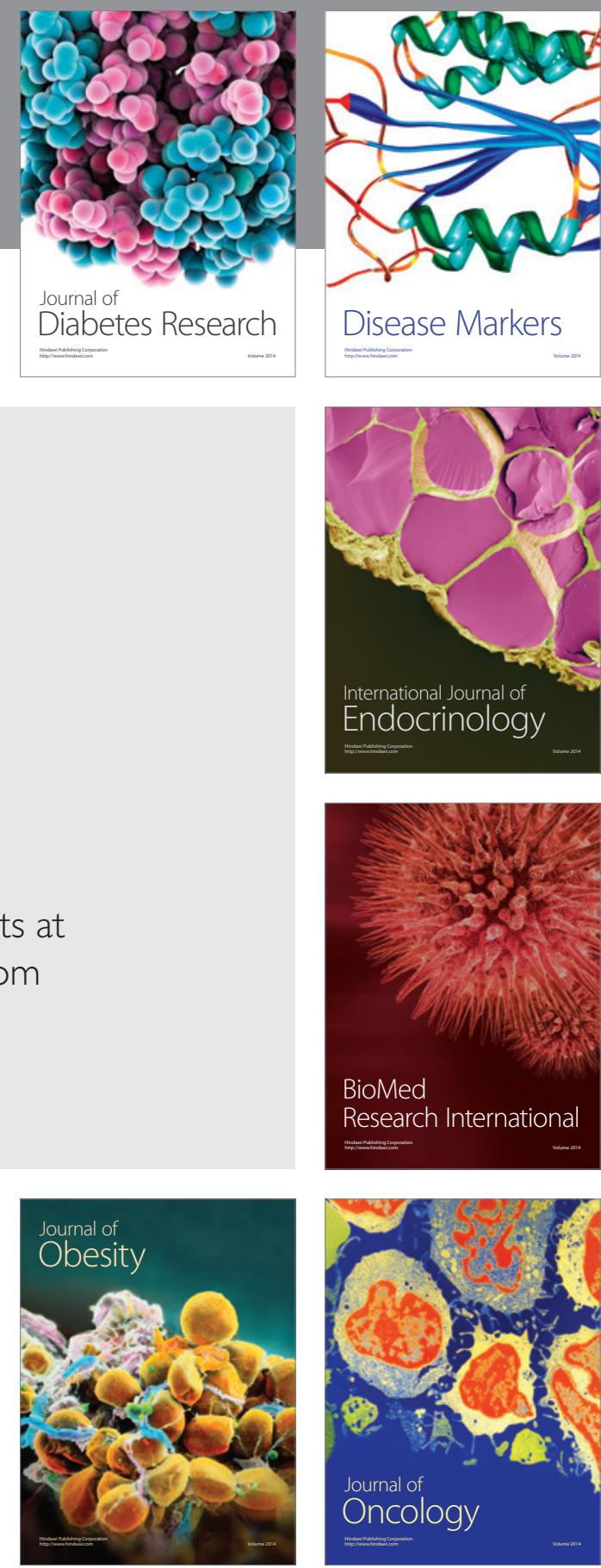

Disease Markers
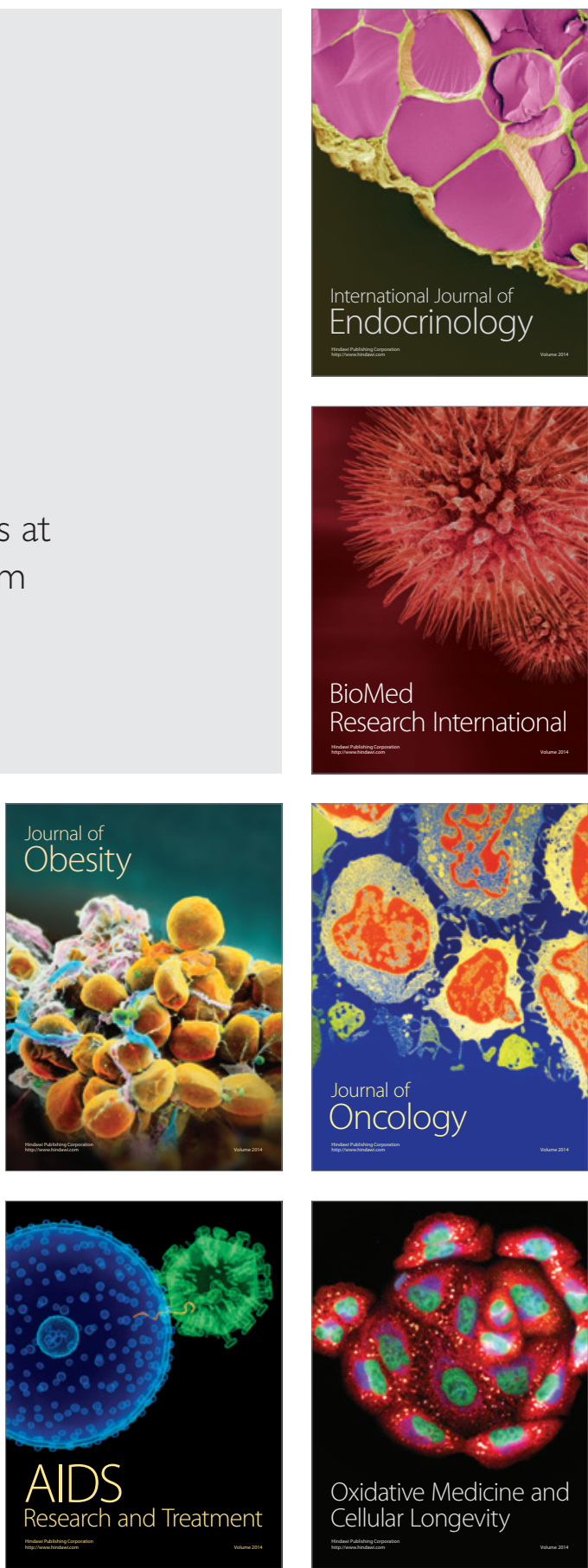